\title{
A Proof on the Conjecture of Twin Primes
}

\section{Zhang Yue}

Department of Physics, Hunan Normal University, Changsha, China

\section{Email address:}

phys_zhangyue a $126 . c 0 m$

\section{To cite this article:}

Zhang Yue. A Proof on the Conjecture of Twin Primes. International Journal of Applied Mathematics and Theoretical Physics. Special Issue: Mathematics Teaching. Vol. 5, No. 3, 2019, pp. 82-84. doi: 10.11648/j.ijamtp.20190503.15

Received: July 15, 2019; Accepted: July 21, 2019; Published: September 20, 2019

\begin{abstract}
Although the mathematicians all over the world offered hard explorations of more than one hundred years, the proof of using pure mathematical theories on the conjecture of twin primes has not born in the world. This paper is trying to apply computer program to prove that corresponding to infinite primes $p$, there are infinite $p+2$ primes. As a mathematical proof, the paper uses the concept of mapping to connect the computer program and the pure mathematical theory. With the requirement of a mathematical proof, in accord with the restriction of the integer of which the computer allows to take, an assumption is suggested, and on the basis of it, using the program of $\mathrm{C}$ language the paper presents, or regarding the $\mathrm{C}$ program as the mapping from infinite $p$ primes to infinite $p+2$ primes, the paper proves that corresponding to infinite primes $p$, there are infinite $\mathrm{p}+2$ primes; namely, the conjecture of twin primes is true.
\end{abstract}

Keywords: Conjecture of Twin Primes, Mapping, Assumption, Program of C Language

\section{Introduction}

Today, there are two difficult problems which have not been solved in the field of the number theory, one of them is the Goldbach conjecture in case of even numbers, and the other is in case of the conjecture of twin primes [1]. The conjecture of twin primes as the 8 th unsolved mathematical problem was proposed in the conference of the mathematicians over the world in 1900 years. During the more than one hundred years, it can be found from some mathematical journals that the conjecture of twin primes as an important topic has been attracting the exciting interests of a lot of researchers who work in the fields of mathematics over the world [2-13]. Up till now, rigorous and satisfactory proof on this conjecture of using the purely mathematical theories has not yet born in the world. The present work is trying to prove the conjecture of twin primes by means of a computer program designed with $\mathrm{C}$ language.

If $Q$ (p) represents a set consisting of infinite primes $\mathrm{p}$, and $\mathrm{Q}(\mathrm{p}+2)$ represents a set consisting of the corresponding infinite $p+2$ primes, assuming that the numbers of bit of binary codes of the computer are not restricted, thus, all the elements of $Q(p+2)$ can be obtained from all of the elements of $\mathrm{Q}(\mathrm{p})$ by executing the $\mathrm{C}$ program. From the viewpoint of mathematics, executing the $\mathrm{C}$ program is identical with a mapping from $\mathrm{Q}(\mathrm{p})$ to $\mathrm{Q}(\mathrm{p}+2)$ [14], it notes $\mathrm{M}: \mathrm{Q}(\mathrm{p}) \rightarrow$
$\mathrm{Q}(\mathrm{p}+2)$.

\section{Assumption}

The computer converts integers into binary codes, if the numbers of bit of the codes are not restricted, the integer $\mathrm{k}$ can be infinitely converted into the binary codes. Nevertheless, the scale of all the computers is limited, the numbers of bit of binary codes of the computer are finite. With respect to the binary codes represented by floating-point machine, if the numbers of bit of exponent number and mantissa of the code are respectively $\mathrm{k}$ bits and $\mathrm{m}$ bits, the range of values taken by integer $\mathrm{N}$ is written: $2^{-2^{k}} \leq \mathrm{N} \leq 2^{\left.2^{k}-1\right)} \cdot\left(1-2^{-\mathrm{m}}\right)$. If the value of $\mathrm{N}$ overpasses this range, it will result in "machinery zero" or "overflow". Proving the conjecture of twin primes requires to consider infinite primes $\mathrm{p}$, but whether $\mathrm{p}+2$ numbers are primes, we are unable to judge all of them one by one by use of the so-called "Exhaustive Attack method". If employing the idea of mathematical induction, certain assumption must be suggested.

Because binary computations of the computer merely relate to logical circuit or Boolean algebra, the rule and result of computations don't change depending up on that there are how many bits of the binary codes. Therefore, it is reasonable to suggest an assumption as follows. 
Assumption: The maximum value of integers limited by the computer is known as $n, p$ is a set consisting of infinite primes $\mathrm{p}_{1}<\mathrm{p}_{2}<\ldots \mathrm{p}_{\mathrm{n}} \ldots$. Arbitrarily considering an integer $\mathrm{k}$ $(\mathrm{k}<\mathrm{n}), \mathrm{R}(\mathrm{k})=\left\{\mathrm{p}_{\mathrm{r}}+2\right\}\left(\mathrm{r}=1,2,3, \ldots \mathrm{i} ; \mathrm{p}_{\mathrm{i}}+2 \leq \mathrm{k}\right)$ represents a set consisting of all the $\mathrm{p}+2$ primes from number 1 to number I which are equal or smaller than $\mathrm{k}$, if $\mathrm{R}(\mathrm{k}+1)=\left\{\mathrm{p}_{\mathrm{t}}+2\right\}(\mathrm{t}=$ $\left.1, \ldots, \mathrm{j} ; \mathrm{p}_{\mathrm{j}}+2 \leq \mathrm{k}+1\right)$ is a set consisting of all the $\mathrm{p}+2$ primes from number 1 to number $\mathrm{j}$ which are equal or smaller than $\mathrm{k}+1(\mathrm{k}+1 \leq \mathrm{n})$, if $\mathrm{j} \geq \mathrm{i}$ holds true for ever, it demonstrates that the set consisting of all the $\mathrm{p}+2$ primes is an infinite set.

In the assumption, $\mathrm{R}(\mathrm{k}+1) \supset \mathrm{R}(\mathrm{k}), \mathrm{R}(\mathrm{k}+1)-\mathrm{R}(\mathrm{k})=\mathrm{k}+1$, $\mathrm{R}(\mathrm{k}+1)$ is more than $\mathrm{R}(\mathrm{k})$ merely one integer $\mathrm{k}+1$. If the range of the integer values taken by the computer is not restricted, $\mathrm{k}$ can be assumed as arbitrarily big, but ii is impossible to occur $\mathrm{j} \equiv \mathrm{I}$ when the value of $\mathrm{k}$ is increased up to some big number, because there are infinite primes $\mathrm{p}$, the opportunities of becoming primes for arbitrary $\mathrm{k}+1$ integers are infinite, therefore, there are infinite cases of $\mathrm{j}$ $\geq \mathrm{i}$.

\section{Proving the Conjecture of Twin Primes}

If $p$ is a prime, and $p+2$ is also a prime, thus, $(p, p+2)$ is called a twin primes, for example, $(5,7), 156 \times 5^{202} \pm 1$, $1159142985 \times 2^{2304} \pm 1$ [15]. In the number theory, the Goldbach conjecture in case of twin primes can be stated as the following theorem.

Theorem: There are infinite primes $p$ to make $p+2$ also become primes.

Designing a program in accord with the theorem, at first, assuming $\mathrm{p}$ is an arbitrary prime, then to find out all the primes from all of the $\mathrm{p}+2$ integers, and proving that the numbers of all the $\mathrm{p}+2$ primes are infinite. The source codes of the program in $\mathrm{C}$ language are presented as follows:

/*Proving the conjecture of twin primes*/

[prime.c Source codes]

\# include $<$ stdio. $\mathrm{h}>$

\# include $<$ math. $\mathrm{h}>$

\# define TRUE 1

\# define FALSE 0

type def int Bool;

Bool is prime (int $n$ );

void main ()

\{

int $\mathrm{p}$;

long int max;

printf ("input a scale max: \n");

scanf ("\%d", \&max);

for $(\mathrm{p}=2 ; \mathrm{p}+2<=\max ; \mathrm{p}++)$

\{

if (is_prime(p)) /*To judge p being primes*/

\{

$\mathrm{p}=\mathrm{p}+2 ; / *$ To find out twin primes $* /$

if (is_prime(p)) /*To judge $\mathrm{p}+2$ being primes*/

printf (“\%d $\% \mathrm{t} ", \mathrm{p}) ; / *$ Out put all of $\mathrm{p}+2$ primes*/

\}

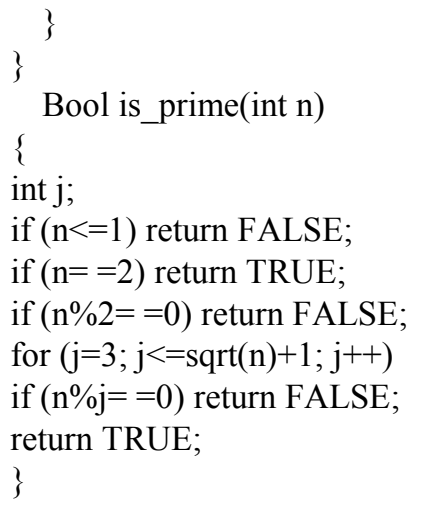

Arbitrarily considering a positive integer within the range of values restricted by the computer, for example, taking max $=\mathrm{k}=100$, executing program prime.c in the platform of C-Free5CJY, the result demonstrates that if $\mathrm{p}$ are primes, all the corresponding $\mathrm{p}+2$ primes of not more than 100 are written:

7, 13, 19, 31, 43, 61, 73 .

In fact, integers 5 and 3 are also a twin primes, corresponding to prime 3,5 is a $p+2$ prime, but it does not appear in the result. The reason is corresponding to $\mathrm{p}+2$ prime 7,5 is a prime, therefore, 5 and 7 can not appear in $\mathrm{p}+2$ primes in the meanwhile.

The similar cases may be very often occurring in the results of executing prime.c for arbitrary max, but those cases don't influence proving that there are infinite $\mathrm{p}+2$ primes.

Then, when $\max =\mathrm{k}+1=101$, executing prime.c in the platform of C-Free $5 \mathrm{CJY}$, the $\mathrm{p}+2$ primes of not more than 101 are also

$7,13,19,31,43,61,73$

This is the case of $\mathrm{j}=\mathrm{i}$ described in the assumption.

If taking $\max =\mathrm{k}=102$, the result of executing prime.c is still the same as that of $\max =k=100$. But when $\max =k+1$ $=103$, executing program prime.c in the platform of $\mathrm{C}$-Free 5 CJY, obtaining $\mathrm{p}+2$ primes:

$7,13,19,31,43,61,73,103$,

This is the case of $\mathrm{j}>\mathrm{i}$ in the assumption. If the range of integers of the computer is not restricted. Executing program prime.c in the platform of C-Free $5 \mathrm{CJY}$ for arbitrarily big integers $\mathrm{k}$ and $\mathrm{k}+1$ can be infinitely realized.

Therefore, according to the assumption, the results of executing prime.c in the platform of C-Free $5 \mathrm{CJY}$ demonstrate that with respect to infinite primes $\mathrm{p}$, from the mapping: $\mathrm{Q}(\mathrm{p}) \rightarrow \mathrm{Q}(\mathrm{p}+2)$, we can find that there are infinite $p+2$ numbers which are also primes. The theorem is proven.

\section{References}

[1] Chen Jing-Run. On some problems in prime number theory [C]. Paris: Seminaire de Theorie des Nombnes, 1979-1980: 167-170.

[2] Brian Conrey and Jonathan P. Keating. Pair Correlations and Twin Primes Revisited [J]. Proc. Math. Phys. Eng. Sci., 2016, 472 (2194): 20160548. 
[3] Zhou Zuo-Ling. A Proof of the Conjecture on the Twin Primes [C]. AIP Conference Proceedings, 2016, 1738 (1): 260002.

[4] Hayat Rezgui. Conjecture of Twin Primes (Still Unsolved Problem in Number Theory). An Expository Essay [J]. Surveys in Mathematics and Its Applications, 2017, 12: 229-252.

[5] Renato Betti. The Twin Primes Conjecture and Other Curiosities Regarding Prime Numbers [J]. Lettera Matematica, 2017, 5 (4): 297-303.

[6] T. J. Hoskins. Proofs of the Twin Primes and Goldbach Conjectures [J]. arXiv. 1901.09668v7 [Math. GM] (e-print), 2019, 7: 1-33.

[7] Andri Lopez. Twin Primes Conjecture and Two Problem More [J]. International Journal of Mathematics and Computation, 2018, 29 (4): 63-66. 107 (1): 55-56.

[8] Maria Suzuki. Alternative Formulations of the Twin Prime Problem [J]. The American Mathematical Monthly, 2000, 107 (1): 55-56.
[9] Juan G. Orozco. An Algorithmic Proof of the Twin Primes Conjecture and the Goldbach Conjecture, viXra.org $>$ number theory > viXra. 1701.0618 [v4], 2018-01-30.

[10] Dieter Sengschmitt. Proof on the Twin Prime Conjecture [J]. viXra.org, Number Theory, viXra: 1710.0042, 2017-10-03.

[11] M. Ram Murty and Akshaa Vatwani. Twin Primes and the Prity Problem [J]. Journal of Number Teory, 2017, 180: 643-659.

[12] Stephan Ramon Garcia, Elvis Kahoro and Florian Luca. Primitive Root Bias for Twin Primes [J]. Experimental Mathematics, 2019, 28 (2): 151-160.

[13] Ramon Ruiz. About the Twin Primes Conjecture [J]. viXra.org, Number Theory, viXra: 1709.0417 [v1], 2017-09-28.

[14] The Mathematical Society of Japan. The Dictionary of Mathematical Encyclopedias (Translation in Chinese) [M]. Beijing: Science Press, 1984: 42-45.

[15] Guy Richard. Unsolved Problems in Number Theory Volume I (M). New York: Springer-Verlag, New York Inc., 1981: 13. 